\title{
Correction to: Selective episiotomy vs. implementation of a non-episiotomy protocol: a randomized clinical trial
}

\author{
Melania M. Amorim ${ }^{1,2^{*}}$, Isabela Cristina Coutinho ${ }^{1}$, Inês Melo ${ }^{1}$ and Leila Katz ${ }^{1}$
}

\section{Correction}

Unfortunately, the original version of this article [1] contained an error. One of the author's full names was not included properly. It is included here correctly. The name of the author is Melania M. Amorim.

Received: 29 September 2017 Accepted: 17 October 2017

Published online: 24 October 2017

\section{Reference}

1. Amorim et al., Selective episiotomy vs. implementation of a non-episiotomy protocol: a randomized clinical trial. Reproductive Health (2017) 14:55 DOI:10.1186/s12978-017-0315-4

\footnotetext{
* Correspondence: melania.amorim@gmail.com

${ }^{1}$ Instituto de Medicina Integral Prof. Fernando Figueira, Women's Healthcare Centre, Recife, Pernambuco 50070-550, Brazil

${ }^{2}$ Instituto Paraibano de Pesquisa Professor Joaquim Amorim Neto, Rua Neusa Borborema, 300, Santo Antônio, Campina Grande 58406-120, Brazil
} 